Article

\title{
Transcriptome Analysis Reveals the Inducing Effect of Bacillus siamensis on Disease Resistance in Postharvest Mango Fruit
}

\author{
Zecheng Jiang ${ }^{1}$, Rui Li ${ }^{2} \mathbb{D}$, Yue Tang ${ }^{1}$, Ziyu Cheng ${ }^{1}$, Minjie Qian ${ }^{2} \mathbb{D}$, Wen Li $^{2, *}$ and Yuanzhi Shao ${ }^{3, *}$ \\ 1 College of Food Science and Engineering, Hainan University, Haikou 570228, China; \\ jiangzecheng516@163.com (Z.J.); ty15828556490@163.com (Y.T.); chengzi_yu@163.com (Z.C.) \\ 2 Key Laboratory for Quality Regulation of Tropical Horticultural Crops of Hainan Province, \\ College of Horticulture, Hainan University, Haikou 570228, China; ruileemo@163.com (R.L.); \\ cookiekin@zju.edu.cn (M.Q.) \\ 3 School of Life Sciences, Hainan University, Haikou 570228, China \\ * Correspondence: liwen9-210@163.com (W.L.); S.YZ123789@163.com (Y.S.); Tel.: +86-13178987302 (W.L.); \\ +86-13005097289 (Y.S.)
}

check for updates

Citation: Jiang, Z.; Li, R.; Tang, Y.; Cheng, Z.; Qian, M.; Li, W.; Shao, Y. Transcriptome Analysis Reveals the Inducing Effect of Bacillus siamensis on Disease Resistance in Postharvest Mango Fruit. Foods 2022, 11, 107. https://doi.org/10.3390/ foods11010107

Academic Editor: Victor Rodov

Received: 14 October 2021

Accepted: 29 December 2021

Published: 1 January 2022

Publisher's Note: MDPI stays neutral with regard to jurisdictional claims in published maps and institutional affiliations.

Copyright: (C) 2022 by the authors. Licensee MDPI, Basel, Switzerland. This article is an open access article distributed under the terms and conditions of the Creative Commons Attribution (CC BY) license (https:// creativecommons.org/licenses/by/ $4.0 /)$.

\begin{abstract}
Postharvest anthracnose, caused by the fungus Colletotrichum gloeosporioides, is one of the most important postharvest diseases of mangoes worldwide. Bacillus siamensis (B. siamensis), as a biocontrol bacteria, has significant effects on inhibiting disease and improving the quality of fruits and vegetables. In this study, pre-storage application of $B$. siamensis significantly induced disease resistance and decreased disease index (DI) of stored mango fruit. To investigate the induction mechanisms of $B$. siamensis, comparative transcriptome analysis of mango fruit samples during the storage were established. In total, 234,808 unique transcripts were assembled and 56,704 differentially expressed genes (DEGs) were identified by comparative transcriptome analysis. Gene ontology (GO) enrichment and Kyoto Encyclopedia of Genes and Genomes (KEGG) pathway analysis of DEGs showed that most of the DEGs involved in plant-pathogen interaction, plant hormone signal transduction, and biosynthesis of resistant substances were enriched. Fourteen DEGs related to disease-resistance were validated by qRT-PCR, which well corresponded to the FPKM value obtained from the transcriptome data. These results indicate that $B$. siamensis treatment may act to induce disease resistance of mango fruit by affecting multiple pathways. These findings not only reveal the transcriptional regulatory mechanisms that govern postharvest disease, but also develop a biological strategy to maintain quality of post-harvest mango fruit.
\end{abstract}

Keywords: Bacillus siamensis; mango fruit; disease resistance; transcriptome analysis; gene expression

\section{Introduction}

Mango fruit (Magnifera indica L.) is one of the most popular fruits with delicious taste, rich nutrition, and a variety of bioactive compounds [1]. However, mango fruit is highly perishable and susceptible to various pathogenic fungi during postharvest. Anthracnose, caused by Colletotrichum gloeosporioides, can cause losses of up to 30 percent the during storage and transportation of mango fruit [2]. Therefore, control of the postharvest disease of mango fruit is still a major issue for decreasing postharvest losses and accelerating the development of the mango industry. Presently, many control strategies against postharvest diseases have been developed. Some physical technologies, such as postharvest heat treatment and UV-C treatment, have been found to effectively control the postharvest diseases of fruits [3]. Synthetic fungicides including mancozeb, carbendazim, promethanide, and tecto60 have been confirmed to have obvious effects on inhibiting anthracnose in mango fruit [4]. Although these fungicides are effective for controlling pathogens, they do have negative influences on human health and the environment [5]. Therefore, seeking effective, 
safe, and valuable alternative fungicides to control postharvest anthracnose disease in mango is of great significance.

As a biological control strategy, microbial antagonists possess a great potential to fight against phytopathogens and have developed into a promising alternative in postharvest disease management [6]. Bacillus, a genus of bacteria, can form spores (endospores), have strong resistance to external harmful factors, and are widely distributed in soil, water, air, and plants. Bacillus siamensis (B. siamensis), as a biocontrol antagonist, was observed to have significant effects on inhibiting disease, improving the quality of fruits and leafy vegetables [7]. B. siamensis was also confirmed to have biocontrol efficacy against brown spot disease of tobacco [8], and the induced tolerance by B. siamensis to salinity stress was found in wheat [9]. According to the present research, the main mechanism of biological antagonism could be contributed to multiple processes, including producing bacteriostatic substances, competing for nutrition and space with pathogens, secreting extracellular lyase, and so on $[10,11]$.

In recent years, high-throughput sequencing technology (RNA-seq) has developed rapidly, and it could be used to provide a comprehensive analysis of the genome and transcriptome of species and reveal the gene expression of individual organisms in specific developmental stages and specific tissues [12]. RNA-seq has become a tool for studying the molecular mechanisms of regulating various physio-chemistry processes, including fruit quality changes, disease response, and so on [13]. Zhao et al. [14] reported the resistance mechanism of citrus by transcriptome sequencing of Geotrichum citri-auranti. Xu et al. [15] explained the complex defense response induced by Talaromyces rugulosus $\mathrm{O} 1$ infection in grapes by RNA-seq analysis. The results of these studies suggest that many biological pathways, including plant hormones, plant-pathogen interactions, reactive oxygen species (ROS), biological stimuli, hormone signaling, and a range of resistance genes, are involved in plant defense systems.

Previously, we have evaluated the effects of B. siamensis against pathogens of tropical fruits, such as mango and lychee, and explored the possible physiological mechanisms in vivo and in vitro [16]. However, the molecular mechanisms of B. siamensis inducing disease resistance in mango fruit have rarely been investigated. The profiles of transcriptomic and gene expression induced by B. siamensis may be crucial for the enhancement of defense response in mango fruits. Therefore, exploring and understanding the induction mechanisms mediated by $B$. siamensis in harvested fruits is beneficial for us to improve the controlling disease technique. In this study, the effect of B. siamensis treatment on controlling decay index caused by anthracnose in mango fruit stored at $25^{\circ} \mathrm{C}$ was determined. The differential genes and regulatory pathways that related to the disease resistance of mango fruit were identified through transcriptomic analysis. Moreover, the patterns and changes in the expression of key differential genes related to the disease response of mango fruit were investigated.

\section{Materials and Methods}

\subsection{Strain}

Bacillus siamensis (B. siamensis) strain was isolated and identified in our laboratory in 2019. Firstly, appropriate B. siamensis was taken from the inclined plane and cultured in Nutrient Agar (NA) at $28^{\circ} \mathrm{C}$ for $48 \mathrm{~h}$. Then, B. siamensis on NA was extracted into $250 \mathrm{~mL}$ Nutrient Broth (NB). It was cultured at $180 \mathrm{rpm}$ at $28^{\circ} \mathrm{C}$ for $20 \mathrm{~h}$. The number of spores was measured with a blood cell counting plate and the concentration of bacterial suspension was adjusted to $1 \times 10^{8}$ cells $/ \mathrm{mL}$ for the next processing.

\subsection{Plant Materials}

Commercial mature mango cultivar 'Tainong' fruits were harvested from a commercial orchard in Lingshui County, Hainan province, and transported to the laboratory as soon as possible. Three hundred uniform fruits without physical injury were chosen and randomly divided into two groups for soaking with tap water (marked with control) and B. siamensis 
suspension $\left(10^{8}\right.$ cells $/ \mathrm{mL}$, marked with treatment) for $20 \mathrm{~min}$. Polyethylene film bag (0.02 $\mathrm{mm}$ in thickness, Fuxiang, Shenzhen) wrapped fruits were stored for 9 days at the temperature and relative humidity of $25^{\circ} \mathrm{C}$ and $95 \%$, respectively. Of the 150 fruits, 30 were labeled for the observation of disease index (DI) and the rest for sampling. Mixed samples of peel and flesh were collected at $0,3,6$, and 9 days after treatment, with one randomly chosen fruit per biological replicate. All treatments were conducted with three biological replicates.

\subsection{Evaluation of Biocontrol Effect of B. siamensis on Pathogens in Stored Mango Fruit}

According to the proportion of the diseased spot area to the fruit surface area, the mangoes in each group were classified as grade 0 (no diseased spot), grade 1 (diseased spot area $<1 / 10$ ), grade 2 (diseased spot area accounted for $1 / 10 \sim 1 / 5$ ), grade 3 (diseased spot area accounted for $1 / 5 \sim 1 / 2$ ), and grade 4 (diseased spot area $>1 / 2$ ). The disease index (DI) was calculated with following formula:

$$
\begin{aligned}
& \mathrm{DI}=\sum \text { (number of fruits in each disease grade } \times \text { the disease grade) } / \\
& \text { (total number of investigations } \times \text { the highest disease grade). }
\end{aligned}
$$

\subsection{Construction and Sequencing of the Transcriptome}

In the present study, twenty-one RNA-Seq libraries for three biological replicates of each of the four time points $(0 \mathrm{~d}, 3 \mathrm{~d}, 6 \mathrm{~d}$, and $9 \mathrm{~d}$ ) of the two groups (control and B. siamensis) were constructed. Total RNA was extracted using the Trizol reagent kit (Invitrogen, Carlsbad, CA, USA) according to the manufacturer's protocol. RNA quality was assessed on an Agilent 2100 Bioanalyzer (Agilent Technologies, Palo Alto, CA, USA) and checked using RNase free agarose gel electrophoresis.

mRNA was enriched, fragmented, and reverse transcribed into cDNA with random primers. After the second-strand cDNA was synthesized, cDNA fragments were purified with the QiaQuick PCR extraction kit (Qiagen, Venlo, The Netherlands), end repaired, A base added, and ligated to Illumina sequencing adapters. After agarose gel electrophoresis, suitable ligation products were selected as templates for PCR amplification, and the library was sequenced using Illumina novaseq 6000 by Gene Denovo Biotechnology Co. (Guangzhou, China). Three biological replicates were performed.

Fastp (version 0.18.0, Shenzhen, China) was used to further filter out the raw reads containing adapters or low quality bases to obtain a high quality clean read. Then, the unigene expression was calculated and normalized to FPKM (fragment per kilobase of transcript per million mapped reads).

\subsubsection{Unigene Functional Annotation}

BLAST alignment was used to annotate the assembled unigenes. The public databased used for BLAST included the NCBI non-redundant protein database (NR, http:/ / www. ncbi.nlm.nih.gov, accessed on 20 August 2021), Swiss-Prot (http:/ / www.expasy.ch/sprot, accessed on 20 August 2021), COG/KOG (http:/ /www.ncbi.nlm.nih.gov/COG, 20 August 2021), Gene Ontology (GO, http:/ / www.geneontology.org, accessed on 20 August 2021), and the Kyoto Encyclopedia of Genes and Genomes (KEGG, http:/ / www.genome.jp/kegg, accessed on 20 August 2021) database.

\subsubsection{Differentially Expressed Genes (DEG) Analysis}

RNAs differential expression analysis was performed by DESeq2 software between two different groups (and by edgeR between two samples). The genes with the parameter of false discovery rate (FDR) below 0.05 and absolute fold change $\geq 2$ were considered differentially expressed genes. 


\subsubsection{GO Enrichment and Pathway Analysis of DEG}

An online platform named OmicShare tools (http: / / www.omicshare.com/tools, accessed on 15 September 2021) was used to obtain the Gene Ontology (GO) annotation and KEGG annotation of DEGs (CK-3d-vs-BS-3d, CK-6d-vs-BS-6d, and CK-9d-vs-BS-9d). GO terms and KEGG terms with corrected $p$ value $\leq 0.05$ were considered significantly enriched.

\subsection{The Quantitative Real-Time PCR ( $q R T-P C R)$ Analysis}

DEGs related to fruit resistance against pathogen infection were screened out, and the selected genes were verified by quantitative reverse transcription polymerase chain reaction (qRT-PCR). Total RNA was extracted by the CTAB method [17]. First-strand cDNA was synthesized from $1 \mu \mathrm{g}$ RNA using the HiScript II Q RT SuperMix (Novizin Bio, Nanjing, China). The primers were designed using the Primer explorer v5 online website (https:/ / primerexplorer.jp/e/, accessed on 10 December 2020) and are listed in Table 1. The ACTIN gene was used as a reference gene. For each sample, three replicates were performed at a final volume of $10 \mu \mathrm{L}$, which consisted of $3.2 \mu \mathrm{L}$ of ddH2O, $0.8 \mu \mathrm{L}$ of primers, $1 \mu \mathrm{L}$ of cDNA, and $5 \mu \mathrm{L}$ of $2 \times$ Q3 SYBR qPCR Master mix (Tolo Biotech, Shanghai, China). The thermal cycling protocol was $5 \mathrm{~min}$ at $95^{\circ} \mathrm{C}$, and then 40 cycles of $95^{\circ} \mathrm{C}$ for $5 \mathrm{~s}$ and $30 \mathrm{~s}$ at $60^{\circ} \mathrm{C}$ for annealing and extension using a qTOWER3 G Real-Time PCR System (Wacker Biotech $\mathrm{GmbH}$, Jena, Germany). The $2^{-\Delta \Delta \mathrm{CT}}$ method was used to calculate the relative expression of genes in the samples.

Table 1. Primers used in the qRT-PCR analysis.

\begin{tabular}{|c|c|c|}
\hline Primers & Gene Symbol & Sequences \\
\hline ACTIN-F & & AATGGAACTGGAATGGTCAAGGC \\
\hline ACTIN-R & & TGCCAGATCTTCTCCATGTCATCCCA \\
\hline Unigene0007915-F & \multirow{2}{*}{ PR1 } & GCTCTCTTCTTCСССТССТ \\
\hline Unigene0007915-R & & TTCTCGCCATATTTCCCAC \\
\hline Unigene0044045-F & \multirow{2}{*}{ NPR1 } & CTCGGCCATCAGATCTCACA \\
\hline Unigene0044045-R & & GAAGATCGTCACCAGCCATAG \\
\hline Unigene0050535-F & \multirow{2}{*}{$B A K 1$} & CCTAACGGGAGATATTCCTGTCAATGG \\
\hline Unigene0050535-R & & GAGGAGGTGGAGATACTGGAAGAGG \\
\hline Unigene0003608-F & \multirow{2}{*}{ GH3 } & ATCAGGAGGGGAGAGAAAG \\
\hline Unigene0003608-R & & CACAAGGCCACCAGGAGTC \\
\hline Unigene0014220-F & \multirow{2}{*}{ WRKY2 } & CCTAACCGCCGATCAGCCATTG \\
\hline Unigene0014220-R & & TCCAATCAAGAGTTCCAGCAGTAGTTC \\
\hline Unigene0053866-F & \multirow{2}{*}{ WRKY22 } & CTTCAAACAACGACAACAGCCTAAGC \\
\hline Unigene0053866-R & & TTCTGGAACTTGGCAAACCCTCTTC \\
\hline Unigene0027317-F & \multirow{2}{*}{ PTI6 } & GACGACGAAGCCTGTCACCATC \\
\hline Unigene0027317-R & & TGGGTTTCTTCTTTCTGGAGGGTTTG \\
\hline Unigene0049558-F & \multirow{2}{*}{$M P K 3$} & ACTCTTCAGATTACACTGCCGCAATAG \\
\hline Unigene0049558-R & & CTGCCTGGAAATAGAGGTCTTCTGTTC \\
\hline Unigene0019931-F & \multirow{2}{*}{ IDH1 } & CGTCATTACCGGGTTCATCAG \\
\hline Unigene0019931-R & & TCCCTGATTCAACCGTTCCA \\
\hline Unigene0038013-F & \multirow{2}{*}{ CAT } & CACATTCAAGAGGACTGGAGGATTCTG \\
\hline Unigene0038013-R & & CCCAAATCATCAAACAGGAAGGTGAAC \\
\hline Unigene0031800-F & \multirow{2}{*}{ SOD1 } & ACGGCTTCCATATTCACGCTCTTG \\
\hline Unigene0031800-R & & CGGCAATAATGTTACCCAAATCACCAG \\
\hline Unigene0020382-F & \multirow{2}{*}{ SOD2 } & CATCACCAGAAGCACCACCAGAC \\
\hline Unigene0020382-R & & CAGACCTCCGCCGTTGAACTTG \\
\hline Unigene0008516-F & \multirow{2}{*}{$P A L$} & CTCCGTCAAGAACTGCGTCACC \\
\hline Unigene0008516-R & & GGTCGTCGGCATAGCTGAACAC \\
\hline Unigene0006190-F & \multirow{2}{*}{$4 C L$} & AAGAGGACGAGAGCCAA \\
\hline Unigene0006190-R & & AGCCGCCCCAGATAATA \\
\hline
\end{tabular}




\subsection{Statistical Analysis}

The data were analyzed by analysis of variance (ANOVA) using the statistical program SPSS/PC version II.x (SPSS Inc. Chicago, IL, USA), and the Duncan's multiple range test was used for mean separation. The statistical significance was assessed at $p \leq 0.05$. Origin 2018 (Origin 2018, Hampton, MA, USA) was used for graphs construction.

\section{Results}

\subsection{The Effect of B. siamensis on Disease Index (DI) of Mango Fruit Stored at $25{ }^{\circ} \mathrm{C}$}

As shown in Figure 1A, B. siamensis treatment significantly slowed down the anthracnose symptoms of mango fruit during the storage. The DI increased during the whole storage period both in the control and in treated fruits (Figure 1B); however, the increase speeds were markedly different between the control and treated fruits, as the DI of $B$. siamensis treated fruits were lower 0.1 and 0.24 than those of control fruit on $6 \mathrm{~d}$ and $9 \mathrm{~d}$, respectively $(p \leq 0.05)$. This indicates that the disease occurrence of mango fruit could be effectively inhibited by $B$. siamensis treatment.

A

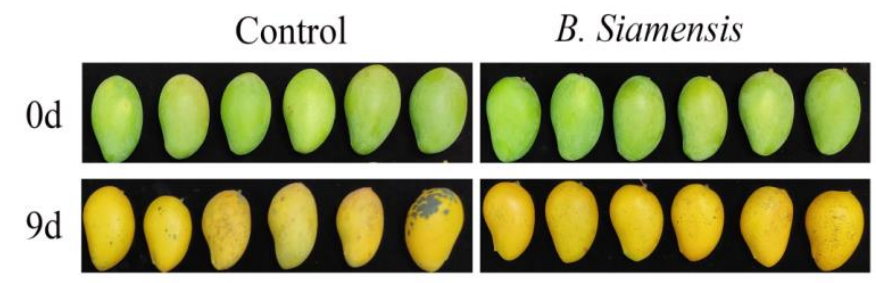

B

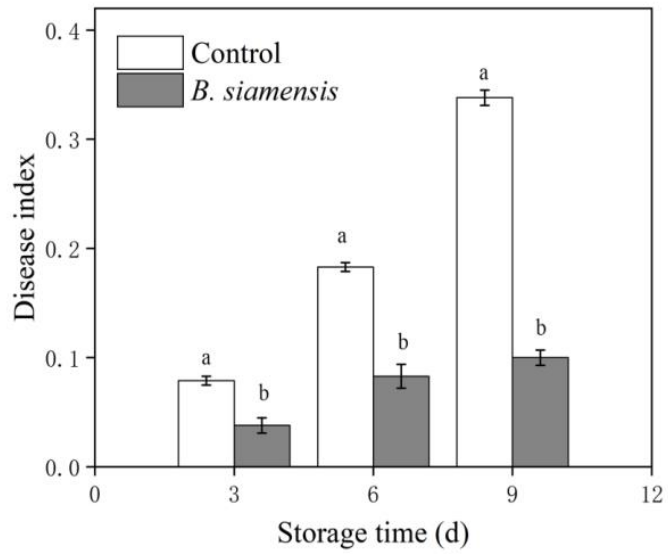

Figure 1. Effect of B. siamensis treatment on appearance and anthracnose symptoms (A) and disease index (B) of mango fruit stored at $25{ }^{\circ} \mathrm{C}$ for 9 days. Bars with different lowercase letters indicate significant differences based on a $t$-test at the $p \leq 0.05$ level. Each value represents the mean $\pm \mathrm{SE}$ of three replicates.

\subsection{Sequencing and Transcriptome Assembly}

A total of $850,798,964$ raw reads were obtained in mango fruit by Illumina HiseqTM 2500 paired-end sequencing. After a stringent filtering process, 839,584,440 higher quality clean reads with $38.9311 \%$ of GC remained (Table 2).

Based on the clean reads, a total of 56,704 unigenes were assembled by the Trinity program. A total of 35,648 (62.87\% of all unigenes) unigenes were annotated (Table 2). 
Table 2. Summary of transcriptome data for mango fruit as well as detailed bioinformatics annotations and analyses.

\begin{tabular}{ll}
\hline Raw Sequences and Assembly Statistics & Number \\
\hline Raw reads (paired-end) & $850,798,964$ \\
clean reads (paired-end) & $839,584,440$ \\
GC content percentage & 38.9311 \\
Total unigenes(average length; N50; min-max length) & $56,704(1114 ; 2058 ; 201-17,696)$ \\
Bioinformatics Annotations of Mango Fruit Unigenes & \\
Gene annotation against Nr (\%) & $35,499(62.60)$ \\
Gene annotation against Swiss-Prot (\%) & $24,644(43.46)$ \\
Gene annotation against KOG (\%) & $20,152(35.54)$ \\
Gene annotation against KEGG (\%) & $33,217(58.58)$ \\
All unigenes annotated (\%) & $35,648(62.87)$ \\
\hline
\end{tabular}

\subsection{Statistics of Different Expressed Genes (DEGs)}

The variation of differential genes in all groups after comparison was shown in Figure 2A. Compared with day 0, there were more and more DEGs in both the treatment group and control group, and there were fewer up-regulated genes than down-regulated ones. For the control fruit, 1890 genes of up-regulation and 4262 genes of down-regulation were observed from day 0 to day 3 (COd-vs-CK-3d). In groups of COd-vs-CK-6d and COdvs-CK-9d, there were much more decreased genes than up-regulated genes. Compared with the control fruit, 2302 genes were up-regulated by B. siamensis treatment on day 3 , and expressions of 4117 and 4681 genes were increased by treatment on day 6 and day 9 , respectively.

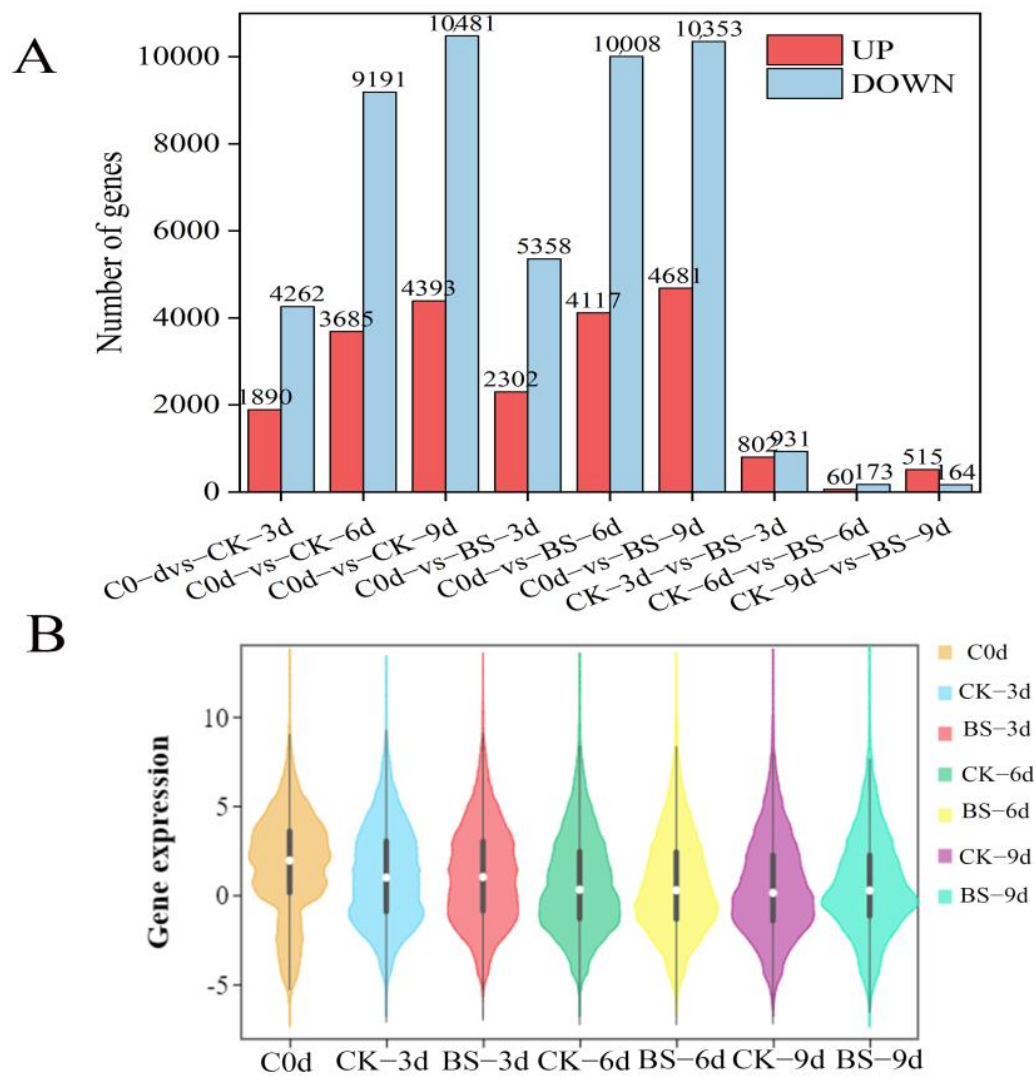

Figure 2. Statistics of differentially expressed genes in all mango fruit groups. (A) Bar chart of the number of all DEGs. (B) Violins in all groups (the white dot in the middle of the box is the median, the upper and lower edge of the box type is $75 \%$, and the upper and lower limit is $90 \%$ ). 
The expression abundance of genes in all samples is presented in Figure 2B. Compared with day 0 , the expression abundance of genes decreased with the extension of storage time. There was no significant difference in overall gene expression between the treatment and control. Because the violin diagram shows the shape of large in the middle and small at the two ends, it indicates that the expression level distribution of day 0 was less uniform than that of other treatment groups. On the third day, both treatment and control expression levels were evenly distributed. The expression levels of all groups had obvious discrete values.

\subsection{GO and KEGG Enrichment Analysis of DEGs in Mango Fruit}

The DEGs of the three comparison groups were collected and then analyzed by GO and KEGG analysis. As shown in Figure 3A, DEGs in the three comparison groups of mangoes can be divided into biological processes, molecular functions, and cellular components, and were further divided into 45 secondary functional groups. Among those groups, 23 subclasses were induced by biological processes. Metabolic process, cell process, and single tissue process were the most abundant of the biological processes. Molecular function includes 11 subclasses, among which catalytic activity and binding activity are the most enriched. Cellular components include 11 subclasses. For cellular components, the organelles were the most abundant among them.

A

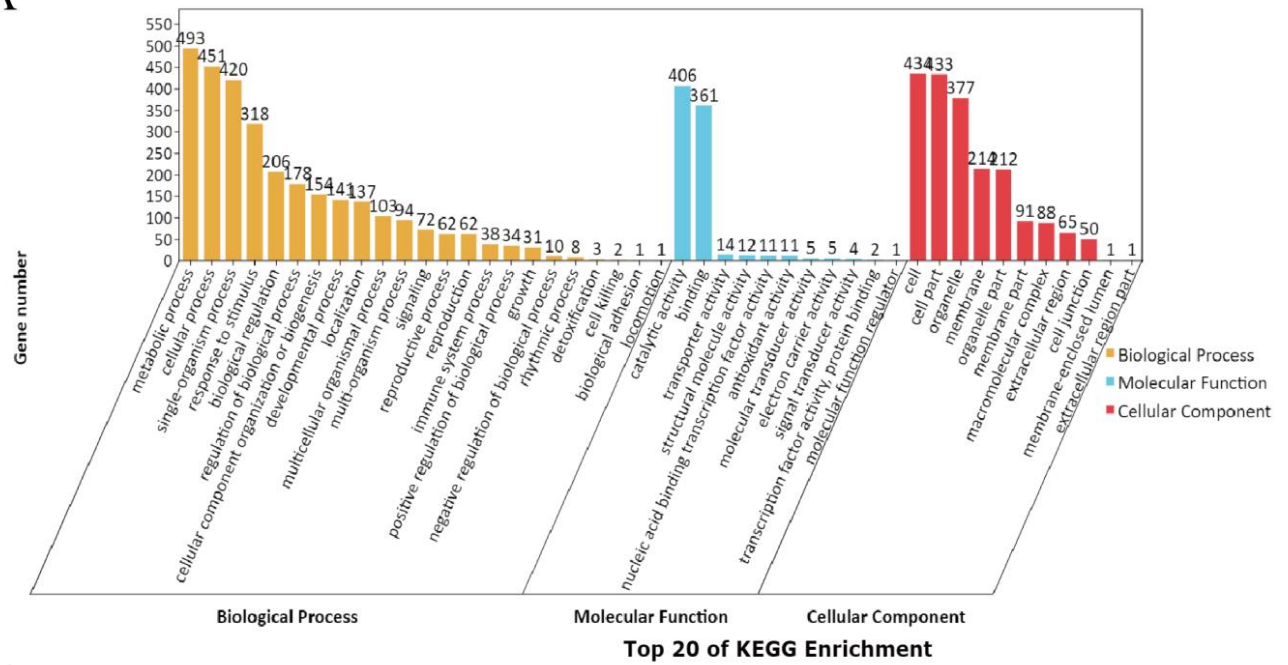

B

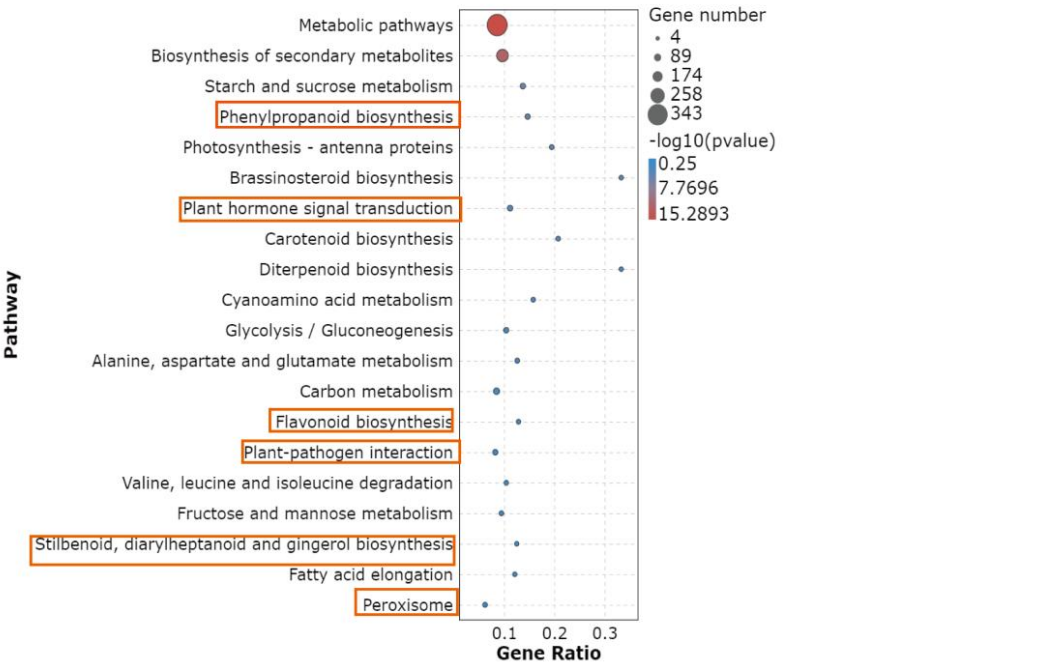

Figure 3. Transcriptomic profiles of mango fruit during storage at $25^{\circ} \mathrm{C}$. (A) GO functional classification of the DEGs. (B) Significant enrichment analysis of KEGG of the DEGs. Six pathways that are associated with disease resistance are highlighted in the orange box. 
The 20 most significantly enriched pathway entries were selected and are shown in Figure 3B. The first three pathways are metabolic pathways, secondary metabolites, and starch and sucrose metabolism. Six pathways that are associated with disease resistance are highlighted in the orange box; they were phenylpropanoid biosynthesis, plant hormone signal transduction, flavonoid biosynthesis, plant-pathogen interaction, stilbenoid, diarylheptanoid and gingerol biosynthesis, and peroxisome pathway.

\subsection{Analysis of Differential Expression Genes (DEGs)}

For comparing the expression difference of key genes between the control and treatment, the following three groups were selected from the total comparison group (Figure 4A): CK-3d-vs-BS-3d (up: 802, down: 931), CK-6d-vs-BS-6d (up: 60, down: 173), and CK-9dvs-BS-9d (up: 515, down: 164). The number of DEGs in different groups was calculated in the histogram (Figure 4A). The CK-3d-vs-BS-3d group had more DEG than the other two groups.

A

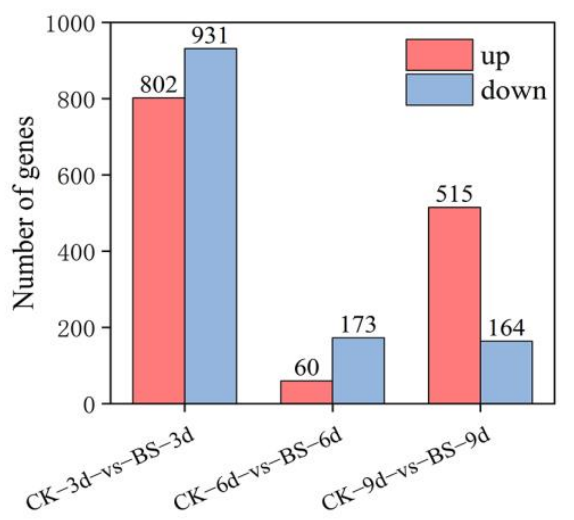

$\mathrm{B}$

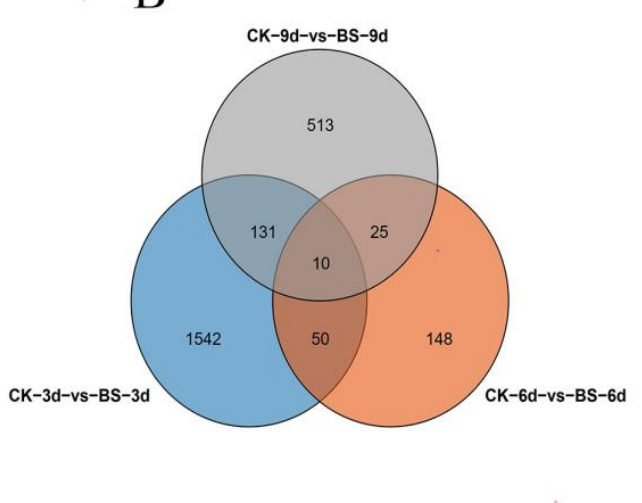

C

CK-3d-vs-BS-3d volcano plot

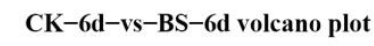

CK-9d-vs-BS-9d volcano plot
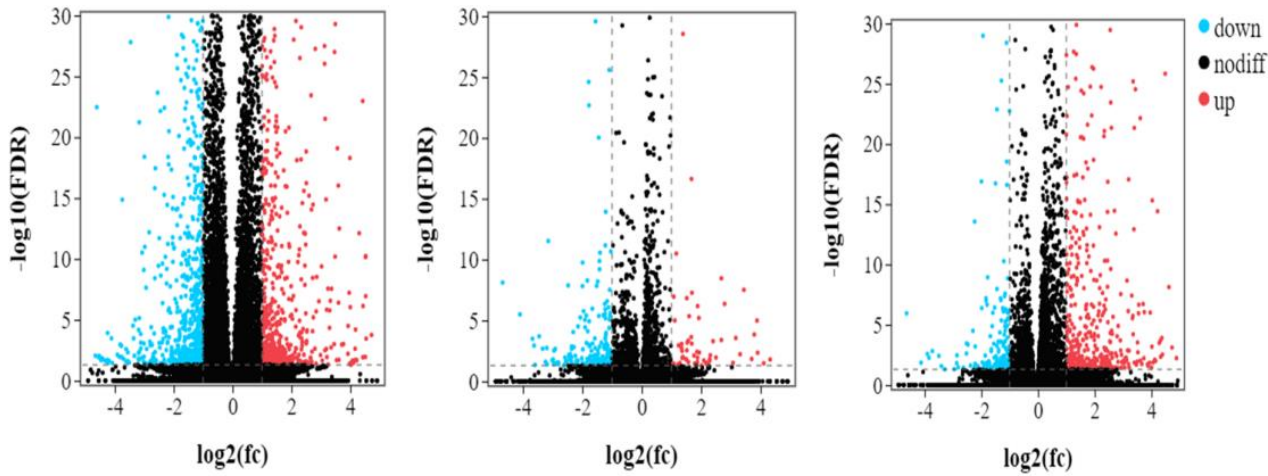

Figure 4. Analyze the different expressed genes in the three comparison mango fruit groups. (A) Bar chart of the number of DEG in three groups. (B) Venn diagram of DEGs among three different comparisons. (C) Volcano plot of DEGs.

Venn diagrams of DEGs in the three comparison groups were generated to show the overlap between the number of DEGs and the comparison groups (Figure 4B). There were 10 overlapping DEGs in all three groups. The CK-3d-vs-BS-3d group and CK-6d-vsBS-6d group had 50 overlapping DEGs, and there were 131 DEGs overlapped with the CK-9d-vs-BS-9d group. CK-6d-vs-BS-6d and CK-9d-vs-BS-9d were overlapped by 25 DEGs.

In the different controls, the volcano plot visually showed the relationship between processing and up-regulation/down-regulation of DEGs number (Figure 4C). We found that in the CK-3d-vs-BS-3d group, the number of DEGs that were up-regulated and down- 
regulated were basically the same. DEGs were mainly up-regulated in the CK-9d-vs-BS-9d comparison group.

\subsection{The Key Genes Analysis in Pathways Related with Disease-Resistance of Mango Fruits}

Six pathways related to the disease-resistance of mango fruits are listed in Supplementary Table S1. The key genes selected in six pathways are exhibited in the Heat map (Figure 5). In plant hormone signal transduction pathway, four genes ( $P R 1, J A Z, B A K 1$, and GH3) were up-regulated and the NPR1 gene in fruits was down-regulated by B. siamensis treatment. In the plant-pathogen interaction pathway, the HSP90A gene was up-regulated and eight genes (WRKY1, WRKY2, WRKY22, PTI5, PTI6, CNGC, FLS2, and MPK3) were down-regulated after 0 days. In the peroxisome pathway, the SOD gene was up-regulated and the $C A T$ gene was down-regulated by $B$. siamensis treatment. In the pathway of phenylpropanoid biosynthesis, $B$. siamensis treatment enhanced the expression of the PAL gene and decreased the expression of the $4 C L$ gene. In flavonoid biosynthesis, two genes (CHS, E5.5.1.6) were down-regulated after 0 days. In the gingerol biosynthesis pathway, HCT and CYP73A were up-regulated by B. siamensis treatment at 9 days.

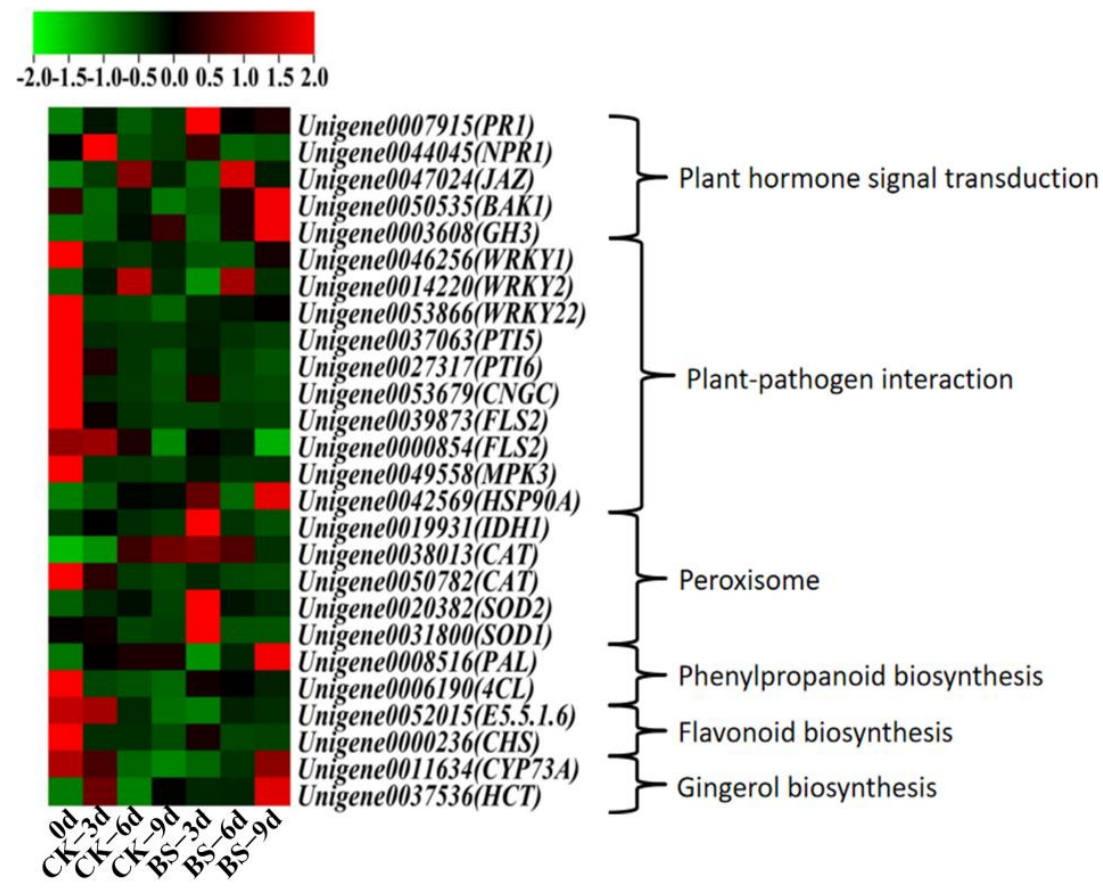

Figure 5. Heat map of gene expression profiles of six disease-resistant pathways that are significantly enriched in stored mango fruits.

\subsection{Validation of Gene Expression Profiles by qRT-PCR}

In order to validate gene expression profiles, 14 unigenes, including genes of $P R 1$, NPR1, JAZ, and GH3, which related to plant hormone signal transduction pathway; genes of WRKY2, WRKY22, PTI6, and MPK3, which related to plant-pathogen interaction pathway; genes of IDH1, CAT, SOD1, and SOD2, which related to peroxisome pathway; and genes of $P A L$ and $4 C L$, which related to phenylpropanoid biosynthesis pathway, were selected for qRT-PCR analysis.

The expression levels of these key genes are shown in Figure 6. In the pathway of plant hormone signal transduction, the expression levels of $P R 1$ and $B A K 1$ were greatly increased by B. siamensis treatment during the whole storage. The expressions of GH3 in treated fruit were distinctly higher than those in the control on days 6 and 9, while expression of NPR1 in treated fruit was higher than that in the control only on day 3. 


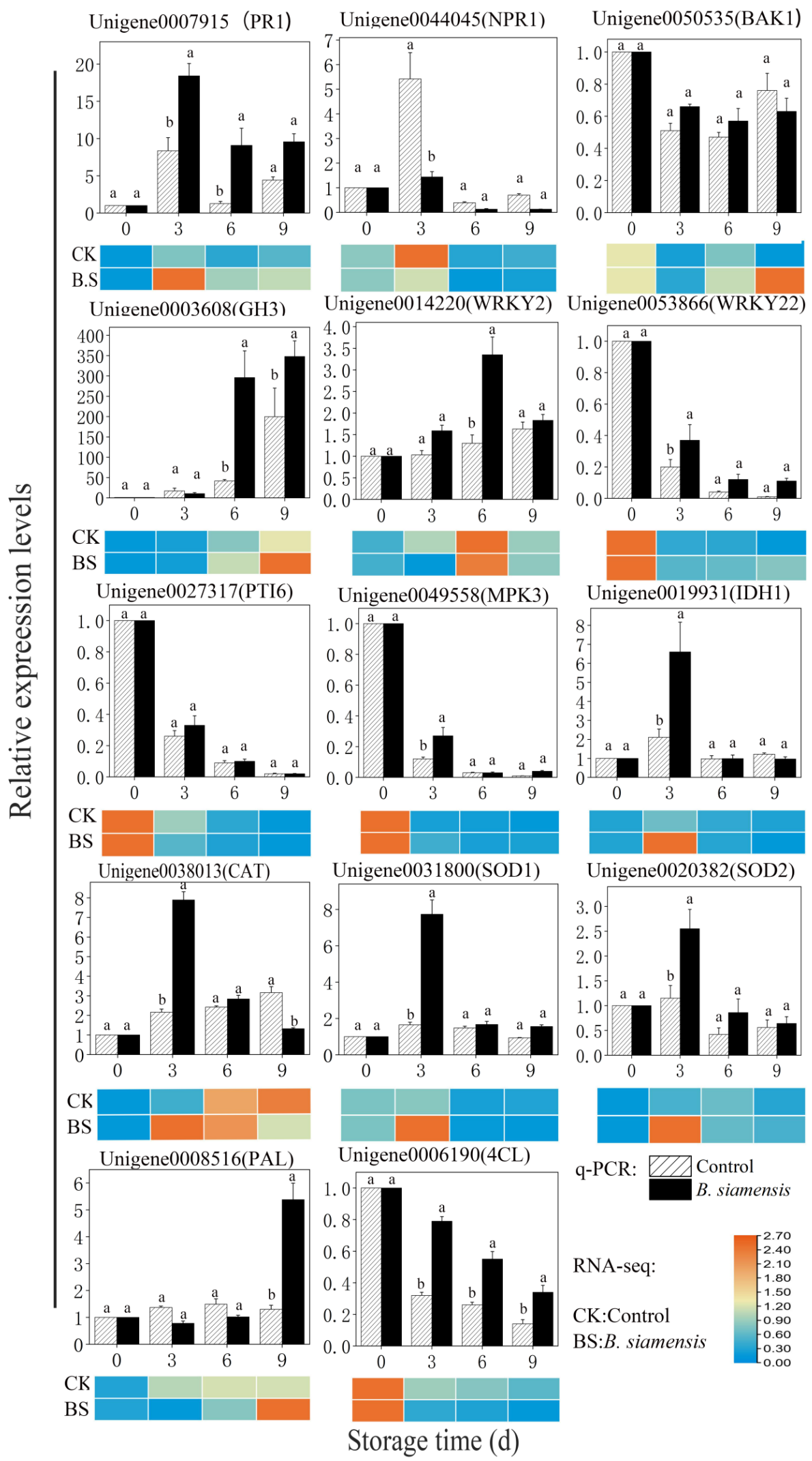

Figure 6. Expression pattern of 14 representative DEGs by qRT-PCR in mango fruits during storage. The expression levels of each unigenes are expressed as a ratio relative to $0 \mathrm{~d}$ of samples, which was set at 1 . The heat map shows the change of the FPKM value of each gene. Error bars indicate standard errors of the means $(n=3)$. Bars with different lower-case letters indicate significant differences based on a $t$-test at the $p \leq 0.05$ level. 
In the plant-pathogen interaction pathway, expressions of WRKY2, WRKY22, PTI6, and MPK3 in the treated fruit were higher than those in the control group at 3,6, and 9 days. For the peroxisome pathway, the significant higher expressions of IDH1, CAT, SOD1, and $S O D 2$ were observed in treated fruits than those of the control fruit during storage of 3 days. In the phenylpropanoid biosynthesis pathway, the expression of the $P A L$ gene in treated fruit was significantly higher than that in the control fruit only on day 9. However, $4 \mathrm{CL}$ gene expressions in treatment group were significantly higher than those in the control group during the whole storage.

FPKM values from transcriptome data are also presented in Supplementary Table S1. Correlation analysis (Supplementary Table S2) showed that the expression levels of genes in PTI6, MPK3, and IDH1 had significant correlations with their FPKM values $\left(\mathrm{R}^{2} \geq 0.99\right)$; they were followed by genes of SOD1, NPR1, and SOD2 $\left(\mathrm{R}^{2} \geq 0.97\right)$. However, no significant relationship between gene expression and FPKM was observed in the WRKY2 gene $\left(\mathrm{R}^{2}=0.534\right)$.

\section{Discussion}

Anthracnose decay is one of the most common and vital fungal diseases of mangoes, and the parameters of DI are regarded as indicators of disease severity in mangoes [18]. In this study, B. siamensis treatment significantly alleviated anthracnose symptoms and reduced the disease index (Figure 1), suggesting that $B$. siamensis treatment enhanced the disease-resistance of stored mango fruits and again confirmed the findings reported in our former studies [16]. Postharvest B. siamensis application also suppressed the development of DI in chickpea and raspberries $[19,20]$. The results suggest that $B$. siamensis could be used as a biological technique to reduce postharvest diseases of fruit and vegetables. Currently, RNA-seq analysis has been employed to study the interactions between antagonistic microorganisms and fruit, and some impressive results have been achieved [21]. Up to now, few studies and reports are available on the molecular response of the mango fruit to B. siamensis.

In our study, compared with control, nearly 2917 genes were significantly affected by B. siamensis, 802 genes were up-regulated by B. siamensis treatment on day 3 , and expressions of 60 and 515 genes were increased by treatment on day 6 and day 9, respectively (Figure 2A). There are more DEGs in mango fruit before 3 days of storage than those of 6 days and 9 days, indicating that the response of the fruit to $B$. siamensis started in the early storage days. There are more up-regulation DEGs than down-regulation DEGs on day 9 (Figure 4A), suggesting that the induced effects of B. siamensis on disease-resistance of fruit could be maintained until the end of storage. In this way, analysis of the possible functions of the DEGs from the comparison between them would help reveal the mechanisms of induced resistance.

Many previous studies demonstrated that genes related to L-phenylalanine metabolism, plant hormone signal transduction, programmed cell death (PCD) regulation, and biosynthesis of amino acids could be induced by antagonistic microorganisms [22,23]. In this study, we found that plant-pathogen interaction, plant hormone signal transduction, and biosynthesis of phenylpropanoid, flavonoid biosynthesis, stilbenoid, diarylheptanoid, and gingerol were the most enriched pathways, whether for control or treatment (Figure 3A,B), indicating that these processes were involved in mango response to B. siamensis.

Plant hormones and phytohormone signaling pathways regulate complex signaling networks associated with plant development and response to environmental stress [24]. An et al. [25] found that 17 genes were enriched in plant hormone signal transduction pathways after being treated with chitosan. PR1 is considered to be a marker of enhanced defense status conferred by pathogen induced systemically acquired resistance (SAR). Yuan et al. [26] found that genes related to PR1 were significantly enriched during fusarium graminearum infection. These findings indicate that the PR1 gene plays an important role in the process of plant and pathogen interaction. Our present study showed that the PR1 gene was significantly up-regulated by B. siamensis treatment (Figure 5). Therefore, 
the increase in anti-disease ability of mango fruit pathogens could be contributed to the induced gene expression by B. siamensis treatment during the storage.

The role of jasmonic acid (JA) in disease resistance of fruit has been widely studied and reported; JA could be rapidly induced to produce an effective defense mechanism when plants are attacked by insects and fungi [27]. As a key regulator of the JA signaling pathway, the $J A Z$ protein plays an important role in plant growth, development, and stress [28]. The $J A Z$ gene is one of the genes encoding the JAZs protein in the TIFY family. The NPR1 gene is another key regulator of salicylic acid (SA) signaling, and it plays an important role in plant defense against pathogens [29]. In this study, the higher expression level of $J A Z$ and the lower expression level of NPR1 in B. siamensis treated fruit than those of control fruit were detected (Figure 5), suggesting that the $J A Z$ gene plays a more important role than the NPR1 gene in disease resistance of stored mango fruit. The BAK1 gene, which regulates the plant immune response [30], was up-regulated by B. siamensis treatment in our study, indicating that the disease resistance of mango could be induced by $B$. siamensis.

In present study, a large number of DEGs in the pathway of plant-pathogen interaction were significantly influenced by $B$. siamensis treatment, suggesting that $B$. siamensis affected the complex signaling networks of stored mango fruit [31]. It has been reported that up-regulation of MPK3 may improve plant resistance by regulating salicylic acid signal transduction [32]. As important modulators of immune response, many members of the plant-specific WRKY transcription factors are related to plant defense against pathogens. It has been found that the expression of WRKY22 was up-regulated in the eliciting resistance of peach fruit to stolon nematodes [33]. In our study, expressions of MPK3 and WRKY22 genes in mango fruit were significantly up-regulated by $B$. siamensis treatment on day 3 of storage and, consequently, the anti-disease ability of fruit induced by $B$. siamensis was enhanced.

In the peroxisome pathway, the accumulation of reactive oxygen species can systematically signal the induction of resistance genes. SOD and CAT are regarded as the enzymes that are most closely related to the metabolism of ROS, and they are dominant in reducing the ROS production, and regulating the balance between the ROS generating and scavenging system may be a way for plants to gain disease resistance [34]. In our study, $B$. siamensis treatment could reduce expression of $C A T$, while increasing the expression of $S O D$, and the increase in $S O D$ activity could enhance the defense against pathogens [35].

In plants, phenylpropionic acid and flavonoid metabolic pathways are considered as two central defense signals under stress [36]. Phenylpropanoid compounds are important secondary metabolites, and these compounds can protect plant from stress damages due to their antioxidant attributes or other protective properties [37]. In the phenylpropanoid biosynthesis pathway, PAL is a key enzyme in the biosynthesis of lignin, salicylic acid, and other phenylalanine metabolism [38]. As a major component of plant cell walls, lignin contributes to the formation of physical and chemical barriers in plant immunity $[39,40]$. In study of pepper fruit, it was found that $P A L$ related genes were activated earlier and changed more in the resistant population than in the susceptible population [41]. Because the phenylpropanoid biosynthesis is the starting point for biosynthesis of several metabolites, activating the phenylpropyl pathway can greatly enhance plant disease resistance [42]. Our present results showed that the expression of the $P A L$ gene induced by $B$. siamensis increased continuously during the storage of mango fruit, while expression of the $P A L$ gene in the control fruit remained stable. The results were consistent with the induction of the PAL gene in avocado fruit reported by Xoca-Orozco et al. [43]. The phenylpropane metabolic pathway is closely related to plant disease resistance. When plants are infected by pathogens, the expressions of $P A L$ and other related genes can be induced, and the increasing in enzyme activity and accumulation of secondary metabolites can prevent the further infection of pathogens, which contribute to all aspects of plant responses towards biotic and abiotic stimuli [44].

Flavonoid pathway is a branch of phenylpropanine metabolism. Flavonoids provide protection by removing reactive oxygen species and limiting the cell death required by pathogens [45]. In the flavonoid pathway, $4 \mathrm{CL}$ is one of the key enzymes to affect the 
biosynthesis of flavonoids and aromatic compounds in plants. In the present study, it was found that the $4 C L$ gene in mango was significantly up-regulated under the induction of $B$. siamensis. CHS is another key gene in the flavonoid pathway, and it was also induced by B. siamensis in mango fruit in our study. Lei et al. [46] reported that the over-expression of CHS is beneficial to the accumulation of rutin and hesperetin. This suggests that the accumulation of flavonoid compound can be increased by up-regulating flavonoid biosynthesis genes, thus improving the resistance of fruits against pathogens.

Another important branch of the phenylpropionic acid defense pathway is the gingerol biosynthesis pathway. Studies have shown that ginger phenol is an important secondary metabolite, which can improve the immune response of plant [47]. In our study, HCT was up-regulated by $B$. siamensis, which was conducive to the accumulation of ferulic acid, the precursor of 6-gingerol, and was beneficial for the induction of disease resistance in stored mango fruit.

\section{Conclusions}

The summary of the mechanisms involved in the disease resistance of mango fruit induced by $B$. siamensis is highlighted in Figure 7 , based on the results of transcriptome analysis. Firstly, some genes $(J A Z, B A K 1$, and PR1) were up-regulated by $B$. siamensis treatment, which triggered a stress response, and induced synthesis of resistant substances of phenols, which improve the disease resistance of mango fruit. Secondly, some genes (WRKY22, HSP90, CNGCS, SOD, PAL, 4CL, CHS, and HCT) related to pathways of plantpathogen interaction, such as peroxisome, phenylpropanoid, flavonoid, and gingerol biosynthesis, were up-regulated by B. siamensis in mango fruit, which enhance the system's anti-disease ability, stimulate the immune response, and, finally, greatly enhance the disease resistance of mango fruit against pathogens.

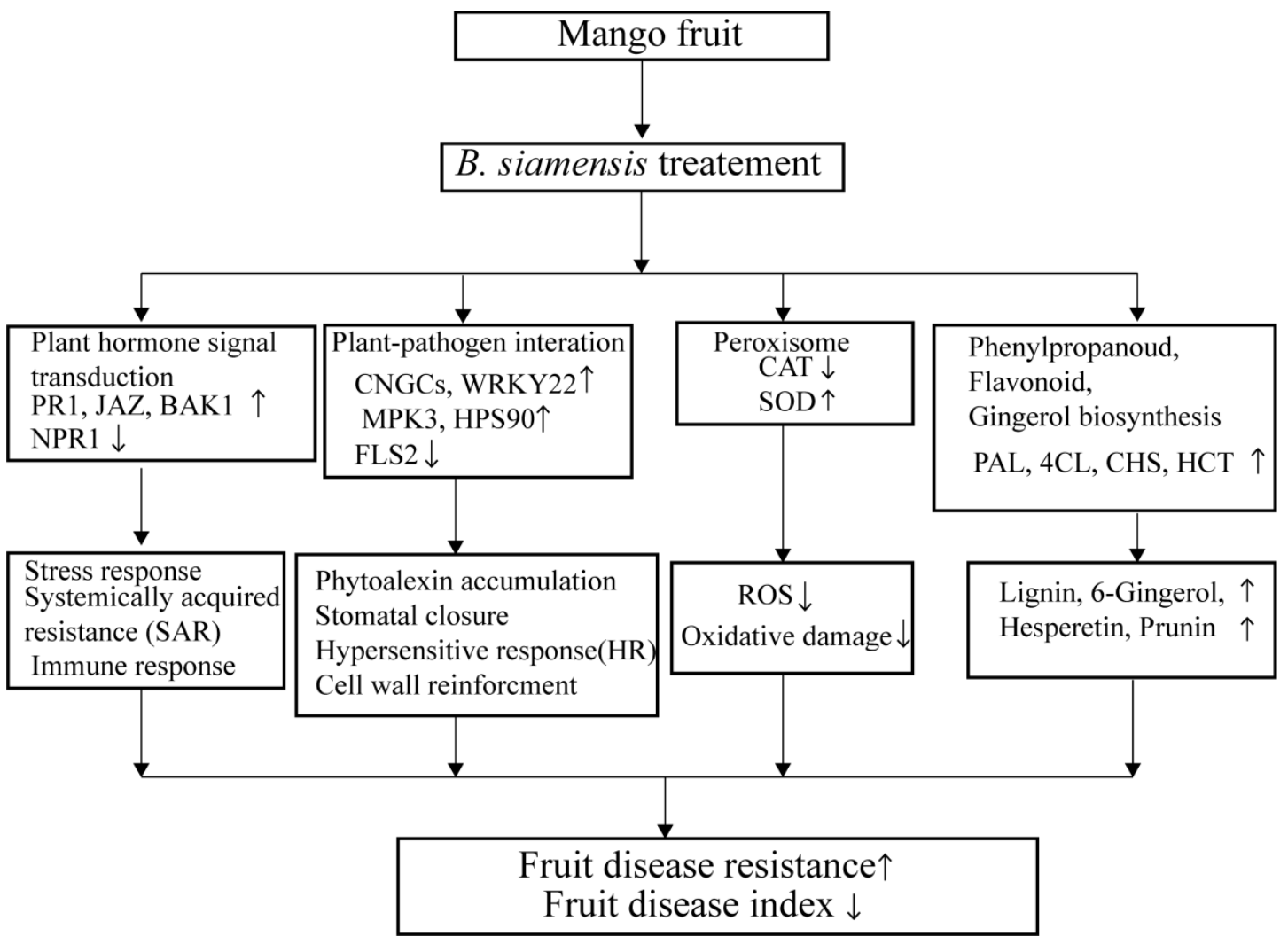

Figure 7. Mechanism diagram of mango defense response to B. siamensis. Through several branched and multi-component pathways, mangoes defense-related genes are transcribed.The up-arrow indicates the promoting effect and the down-arrow indicates the inhibiting effect. 
Overall, the disease resistance of mango fruit could be improved by B. siamensis. The results of this research provide a reference for future studies on controlling postharvest disease of fruit and vegetables using antagonistic bacteria and provide a theoretical basis for the practical application of an antagonistic agent.

Supplementary Materials: The following are available online at https:/ /www.mdpi.com/article/10 .3390 / foods11010107/s1, Table S1: Expression and description of selected resistance genes at four $(0,3,6,9)$ different time points. Table S2: Correlation between gene expression and FPKM value. Significant correlation: $\mathrm{R}^{2} \geq 0.98$, No correlation: $\mathrm{R}^{2}<0.5$.

Author Contributions: Y.S., W.L. conceived and designed the experiments. Z.J., Y.T. and Z.C. performed the experiments and wrote the paper. R.L. and M.Q. analyzed the data. All authors have read and agreed to the published version of the manuscript.

Funding: This research was funded by National Natural Science Foundation of China (Grant Number 32060564).

Institutional Review Board Statement: Not applicable.

Informed Consent Statement: Not applicable.

Data Availability Statement: Data sharing not applicable.

Conflicts of Interest: The authors declare no conflict of interest.

\section{References}

1. Li, L.; Wu, H.X.; Ma, X.W.; Xu, W.T.; Liang, Q.Z.; Zhan, R.L.; Wang, S.B. Transcriptional mechanism of differential sugar accumulation in pulp of two contrasting mango (Mangifera indica L.) cultivars. Genomics 2020, 112, 4505-4515. [CrossRef] [PubMed]

2. He, J.; Ren, Y.; Chen, C.; Liu, J.; Liu, H.; Pei, Y. Defense Responses of Salicylic Acid in Mango Fruit Against Postharvest Anthracnose, Caused by Colletotrichum gloeosporioides and its Possible Mechanism. J. Food Saf. 2017, 37, e12294. [CrossRef]

3. Zhang, W.; Jiang, H.; Cao, J.; Jiang, W. UV-C treatment controls brown rot in postharvest nectarine by regulating ROS metabolism and anthocyanin synthesis. Postharvest Biol. Technol. 2021, 180, 111613. [CrossRef]

4. Chechi, A.; Stahlecker, J.; Dowling, M.E.; Schnabel, G. Diversity in species composition and fungicide resistance profiles in Colletotrichum isolates from apples. Pestic. Biochem. Physiol. 2019, 158, 18-24. [CrossRef]

5. Sun, C.; Fu, D.; Lu, H.; Zhang, J.; Zheng, X.; Yu, T. Autoclaved yeast enhances the resistance against Penicillium expansum in postharvest pear fruit and its possible mechanisms of action. Biol. Control 2018, 119, 51-58. [CrossRef]

6. Fernandez-San Millan, A.; Larraya, L.; Farran, I.; Ancin, M.; Veramendi, J. Successful biocontrol of major postharvest and soil-borne plant pathogenic fungi by antagonistic yeasts. Biol. Control 2021, 160, 104683. [CrossRef]

7. $\quad$ Feng, F.; Chen, X.; Wang, Q.; Xu, W.; Long, L.; Nabil El-Masry, G.; Wan, Q.; Yan, H.; Cheng, J.; Yu, X. Use of Bacillus-siamensisinoculated biochar to decrease uptake of dibutyl phthalate in leafy vegetables. J. Environ. Manag. 2020, 253, 109636. [CrossRef] [PubMed]

8. Xie, Z.; Li, M.; Wang, D.; Wang, F.; Shen, H.; Sun, G.; Feng, C.; Wang, X.; Chen, D.; Sun, X. Biocontrol efficacy of Bacillus siamensis LZ88 against brown spot disease of tobacco caused by Alternaria alternata. Biol. Control 2021, 154, 104508. [CrossRef]

9. Amna; Ud Din, B.; Sarfraz, S.; Xia, Y.; Kamran, M.A.; Javed, M.T.; Sultan, T.; Hussain Munis, M.F.; Chaudhary, H.J. Mechanistic elucidation of germination potential and growth of wheat inoculated with exopolysaccharide and ACC- deaminase producing Bacillus strains under induced salinity stress. Ecotoxicol. Environ. Saf. 2019, 183, 109466. [CrossRef]

10. Liu, J.; Sui, Y.; Wisniewski, M.; Droby, S.; Liu, Y. Review: Utilization of antagonistic yeasts to manage postharvest fungal diseases of fruit. Int. J. Food Microbiol. 2013, 167, 153-160. [CrossRef]

11. Syed Ab Rahman, S.F.; Singh, E.; Pieterse, C.M.J.; Schenk, P.M. Emerging microbial biocontrol strategies for plant pathogens. Plant Sci. 2018, 267, 102-111. [CrossRef] [PubMed]

12. Wang, Q.-H.; Ji, Y.-P.; Qu, Y.-Y.; Qi, Y.-K.; Li, D.-W.; Liu, Z.-Y.; Wu, X.-Q. The response strategies of Colletotrichum gloeosporioides s.s. due to the stress caused by biological control agent Bacillus amyloliquefaciens deciphered by transcriptome analyses. Biol. Control 2020, 150, 104372. [CrossRef]

13. Yang, R.; Lin, X.; Dou, Y.; Zhang, W.; Du, H.; Wan, C.; Chen, J.; Zhang, L.; Zhu, L. Transcriptome profiling of postharvest kiwifruit in response to exogenous nitric oxide. Sci. Hortic. 2021, 277, 109788. [CrossRef]

14. Zhao, J.; Zhang, D.; Wang, Z.; Tian, Z.; Yang, F.; Lu, X.; Long, C.A. Genome sequencing and transcriptome analysis of Geotrichum citri-aurantii on citrus reveal the potential pathogenic- and guazatine-resistance related genes. Genomics 2020, 112, $4063-4071$. [CrossRef]

15. Xu, M.; Zhang, X.; Li, D.; Gu, X.; Godana, E.A.; Dhanasekaran, S.; Zhao, L.; Zhang, H. Transcriptome analysis of postharvest grapes in response to Talaromyces rugulosus O1 infection. Postharvest Biol. Technol. 2021, 178, 111542. [CrossRef] 
16. You, W.; Ge, C.; Jiang, Z.; Chen, M.; Li, W.; Shao, Y. Screening of a broad-spectrum antagonist-Bacillus siamensis, and its possible mechanisms to control postharvest disease in tropical fruits. Biol. Control 2021, 157, 104584. [CrossRef]

17. Palani, S.N.; Elangovan, S.; Menon, A.; Kumariah, M.; Tennyson, J. An efficient nucleic acids extraction protocol for Elettaria cardamomum. Biocatal. Agric. Biotechnol. 2019, 17, 207-212. [CrossRef]

18. Hong, K.; Gong, D.; Zhang, L.; Hu, H.; Jia, Z.; Gu, H.; Song, K. Transcriptome characterization and expression profiles of the related defense genes in postharvest mango fruit against Colletotrichum gloeosporioides. Gene 2016, 576, 275-283. [CrossRef] [PubMed]

19. Gorai, P.S.; Ghosh, R.; Mandal, S.; Ghosh, S.; Chatterjee, S.; Gond, S.K.; Mandal, N.C. Bacillus siamensis CNE6-A multifaceted plant growth promoting endophyte of Cicer arietinum L. having broad spectrum antifungal activities and host colonizing potential. Microbiol. Res. 2021, 252, 126859. [CrossRef]

20. Zhang, X.; Gao, Z.; Zhang, X.; Bai, W.; Zhang, L.; Pei, H.; Zhang, Y. Control effects of Bacillus siamensis G-3 volatile compounds on raspberry postharvest diseases caused by Botrytis cinerea and Rhizopus stolonifer. Biol. Control 2020, 141, 104135. [CrossRef]

21. Zhao, L.; Zhu, H.; Li, B.; Legrand Ngolong Ngea, G.; Gu, X.; Zhang, X.; Dhanasekaran, S.; Zhang, H. Transcriptomic analysis of the disease-resistance response in mandarins induced by the biocontrol yeast, Yarrowia lipolytica. Biol. Control 2021, $163,104607$. [CrossRef]

22. Christopoulos, M.V.; Tsantili, E. Participation of phenylalanine ammonia-lyase (PAL) in increased phenolic compounds in fresh cold stressed walnut (Juglans regia L.) kernels. Postharvest Biol. Technol. 2015, 104, 17-25. [CrossRef]

23. Postel, S.; Kemmerling, B. Plant systems for recognition of pathogen-associated molecular patterns. Semin. Cell Dev. Biol. 2009, 20, 1025-1031. [CrossRef] [PubMed]

24. Bari, R.; Jones, J.D. Role of plant hormones in plant defence responses. Plant Mol. Biol. 2009, 69, 473-488. [CrossRef] [PubMed]

25. An, N.; Lv, J.; Zhang, A.; Xiao, C.; Zhang, R.; Chen, P. Gene expression profiling of papaya (Carica papaya L.) immune response induced by CTS-N after inoculating PLDMV. Gene 2020, 755, 144845. [CrossRef]

26. Yuan, G.; He, X.; Li, H.; Xiang, K.; Liu, L.; Zou, C.; Lin, H.; Wu, J.; Zhang, Z.; Pan, G. Transcriptomic responses in resistant and susceptible maize infected with Fusarium graminearum. Crop J. 2020, 8, 153-163. [CrossRef]

27. Yang, Y.; Liu, J.; Zhou, X.; Liu, S.; Zhuang, Y. Transcriptomics analysis unravels the response to low temperature in sensitive and tolerant eggplants. Sci. Hortic. 2020, 271, 109468. [CrossRef]

28. Li, C.; Shi, L.; Wang, Y.; Li, W.; Chen, B.; Zhu, L.; Fu, Y. Arabidopsis ECAP Is a New Adaptor Protein that Connects JAZ Repressors with the TPR2 Co-repressor to Suppress Jasmonate-Responsive Anthocyanin Accumulation. Mol. Plant 2020, 13, 246-265. [CrossRef] [PubMed]

29. Wang, Z.; Zhang, W.-H.; Ma, L.-Y.; Li, X.; Zhao, F.-Y.; Tan, X.-L. Overexpression of Brassica napus NPR1 enhances resistance to Sclerotinia sclerotiorum in oilseed rape. Physiol. Mol. Plant Pathol. 2020, 110, 101460. [CrossRef]

30. Yu, X.; Xu, G.; Li, B.; de Souza Vespoli, L.; Liu, H.; Moeder, W.; Chen, S.; de Oliveira, M.V.V.; Ariadina de Souza, S.; Shao, W.; et al The Receptor Kinases BAK1/SERK4 Regulate $\mathrm{Ca}(2+)$ Channel-Mediated Cellular Homeostasis for Cell Death Containment. Curr. Biol. 2019, 29, 3778-3790.e3778. [CrossRef]

31. Elmore, J.M.; Griffin, B.D.; Walley, J.W. Advances in functional proteomics to study plant-pathogen interactions. Curr. Opin. Plant Biol. 2021, 63, 102061. [CrossRef]

32. Long, L.; Xu, F.C.; Zhao, J.R.; Li, B.; Xu, L.; Gao, W. GbMPK3 overexpression increases cotton sensitivity to Verticillium dahliae by regulating salicylic acid signaling. Plant Sci. 2020, 292, 110374. [CrossRef]

33. Li, C.; Wang, J.; Ji, N.; Lei, C.; Zhou, D.; Zheng, Y.; Wang, K. PpHOS1, a RING E3 ubiquitin ligase, interacts with PpWRKY22 in the BABA-induced priming defense of peach fruit against Rhizopus stolonifer. Postharvest Biol. Technol. 2020, 159, 111029. [CrossRef]

34. Qiu, H.; Su, L.; Wang, H.; Zhang, Z. Chitosan elicitation of saponin accumulation in Psammosilene tunicoides hairy roots by modulating antioxidant activity, nitric oxide production and differential gene expression. Plant Physiol. Biochem. 2021, 166, 115-127. [CrossRef] [PubMed]

35. Singh, B.; Kaur, N.; Kumar, P.; Hallan, V.; Pati, P.K. Reactive oxygen species generating and scavenging systems play critical role in conferring leaf spot disease resistance in Withania somnifera (L.) Dunal. Ind. Crop. Prod. 2020, 157, 112889. [CrossRef]

36. Akbarian, A.; Rahimmalek, M.; Sabzalian, M.R.; Hodaei, M. Sequencing and phylogenetic analysis of phenylalanine ammonia lyase (pal) and chalcone synthase (chs) genes in some Iranian endemic species of Apiaceae. Gene Rep. 2021, 23, 112889. [CrossRef]

37. Lu, D.; Zhiqiang, H.; Di, L.; Pengfang, Z.; Shengjin, L.; Na, L.; Hongyu, M. Transcriptome analysis of chrysanthemum in responses to white rust. Sci. Hortic. 2018, 233, 421-430. [CrossRef]

38. You, X.; Fang, H.; Wang, R.; Wang, G.-L.; Ning, Y. Phenylalanine ammonia lyases mediate broad-spectrum resistance to pathogens and insect pests in plants. Sci. Bull. 2020, 65, 1425-1427. [CrossRef]

39. Dehghan, S.; Sadeghi, M.; Poppel, A.; Fischer, R.; Lakes-Harlan, R.; Kavousi, H.R.; Vilcinskas, A.; Rahnamaeian, M. Differential inductions of phenylalanine ammonia-lyase and chalcone synthase during wounding, salicylic acid treatment, and salinity stress in safflower, Carthamus tinctorius. Biosci. Rep. 2014, 34, e00114. [CrossRef]

40. Gharibi, S.; Sayed Tabatabaei, B.E.; Saeidi, G.; Talebi, M.; Matkowski, A. The effect of drought stress on polyphenolic compounds and expression of flavonoid biosynthesis related genes in Achillea pachycephala Rech.f. Phytochemistry 2019, 162, 90-98. [CrossRef]

41. Wang, R.; Wang, G.L.; Ning, Y. PALs: Emerging Key Players in Broad-Spectrum Disease Resistance. Trends Plant Sci. 2019, 24, 785-787. [CrossRef] 
42. Irani, S.; Todd, C.D.; Wei, Y.; Bonham-Smith, P.C. Changes in phenylpropanoid pathway gene expression in roots and leaves of susceptible and resistant Brassica napus lines in response to Plasmodiophora brassicae inoculation. Physiol. Mol. Plant Pathol. 2019, 106, 196-203. [CrossRef]

43. Xoca-Orozco, L.A.; Aguilera-Aguirre, S.; Vega-Arreguin, J.; Acevedo-Hernandez, G.; Tovar-Perez, E.; Stoll, A.; Herrera-Estrella, L.; Chacon-Lopez, A. Activation of the phenylpropanoid biosynthesis pathway reveals a novel action mechanism of the elicitor effect of chitosan on avocado fruit epicarp. Food Res. Int. 2019, 121, 586-592. [CrossRef] [PubMed]

44. Vogt, T. Phenylpropanoid biosynthesis. Mol. Plant 2010, 3, 2-20. [CrossRef]

45. Jiang, L.; Wu, P.; Yang, L.; Liu, C.; Guo, P.; Wang, H.; Wang, S.; Xu, F.; Zhuang, Q.; Tong, X.; et al. Transcriptomics and metabolomics reveal the induction of flavonoid biosynthesis pathway in the interaction of Stylosanthes-Colletotrichum gloeosporioides. Genomics 2021, 113, 2702-2716. [CrossRef]

46. Lei, H.; Niu, T.; Song, H.; Bai, B.; Han, P.; Wang, Z.; Liu, A. Comparative transcriptome profiling reveals differentially expressed genes involved in flavonoid biosynthesis between biennial and triennial Sophora flavescens. Ind. Crop. Prod. 2021, $161,11321$. [CrossRef]

47. Li, R.; Li, Y.; Zhang, Y.; Sheng, J.; Zhu, H.; Shen, L. Transcriptome analysis reveals that SINPR1 mediates tomato fruit resistance against Botrytis cinerea by modulating phenylpropanoid metabolism and balancing ROS homeostasis. Postharvest Biol. Technol. 2021, 172, 111382. [CrossRef] 